\title{
FITTING THE SPREADING DIAMETER OF A GLYCERINE DROPLET IMPACT ONTO HORIZONTAL SURFACES
}

\author{
Aleksandra Kostić, Valentina Timotić, Šefko Šikalo \& Jelena Radović
}
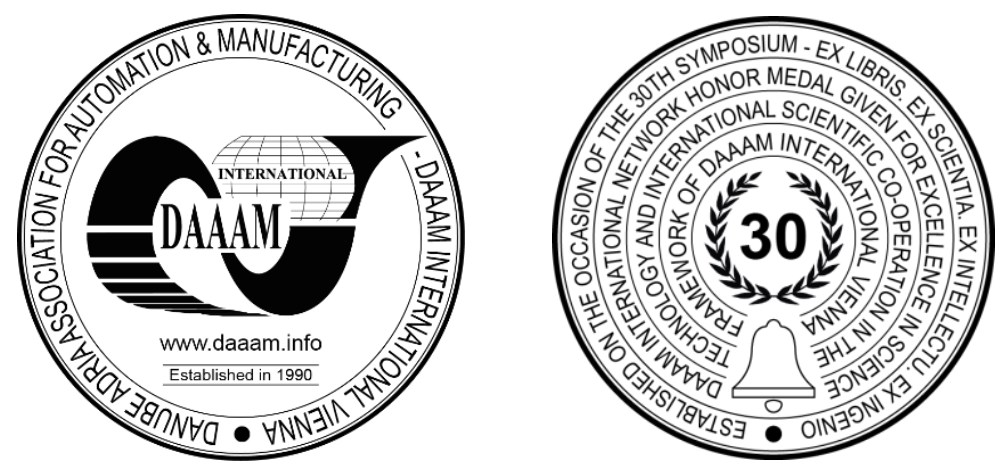

This Publication has to be referred as: Kostic, A[leksandra]; Timotic, V[alentina]; Sikalo, S[efko] \& Radovic, J[elena] (2020). Fitting the Spreading Diameter of a Glycerine Droplet Impact onto Horizontal Surfaces, Proceedings of the 31st DAAAM International Symposium, pp.0943-0949, B. Katalinic (Ed.), Published by DAAAM International, ISBN 978-3-902734-29-7, ISSN 1726-9679, Vienna, Austria

DOI: $10.2507 / 31$ st.daaam.proceedings.131

\begin{abstract}
Dynamic behaviour of liquid droplets has many applications in process engineering, such as spray painting, spray forming, chemical evaporators, coating in wood industry, PVC manufacturing. We will consider glycerine as the representative of the liquids with high viscosity. Unlike water, due to high viscosity, reducing of the diameter is rare and it occurs only with high Weber numbers. Šikalo and Kostić have given an equation for fitting the spreading diameter of a water droplet impacting on flat surfaces, using a rational function. For glycerine, the function for fitting of the data will have two analytical expressions - the rational function and the constant function. Data for the impact of glycerine droplets, with diameter of $2.45 \mathrm{~mm}$, and the Weber numbers 51, 93, 163, 280, 402, 571, 802, 1056 are fitted to the rational and constant functions. We will apply for the coefficient of the rational function the properties of the glycerine droplets and the experimental maximum. The experimental maximum corresponds to a stationary point of rational functions. The constant functions are the experimental maximum and that is due to the high viscosity of glycerine. Numerical experiments indicate good agreement between the experimental data and proposed function.
\end{abstract}

Keywords: Droplet impact; spreading diameter; glycerine; high viscosity.

\section{Introduction}

Knowledge of the dynamics of the impact of droplets on a solid surface is important in the study of phenomena that occur in many natural and industrial processes. Some of these processes are painting and spraying, droplet injection into diesel engines, inkjet printers, metal spray cooling, droplet impact into steam turbine blades, spraying chemicals in agriculture, fire prevention by water spray system, cooling of electronics, metal droplet spraying casting, the impact of droplets on the wing of an airplane when landing during rain, leading to a loss of its performance. Many studies, which belong to experimental, numerical and analytical research have been published. These studies cover a wide range of conditions, for which outcomes need to be assessed. Extensive experimental and numerical research has been published in [2]. In the paper [11] experiments of droplet impact onto dry surfaces of smooth and rough glass, PVC, and wax with different contact angles ranging from $6^{\circ}$ to $105^{\circ}$, are carried out. Glycerine, water and isopropanol droplets are used. The instantaneous spread diameters and contact angles are measured. Numerical simulations are carried out using finite volume method in an unstructured grid. It is found that the wall treatment using an appropriate contact angle model improved the accuracy of the numerical results. 
The experimental data fitted in this paper are published in [2]. Knowledge of the function spreading diameter depending on time, allows comparison with numerical calculations, as well as the estimation of diverse applications of droplet impact in process engineering, which have motivated experimental, theoretical and numerical research of this problem [1], [2], [3], [4], [5], [6], [7], [8], [9], [10]. The dependency of spreading diameter on time is a function of the following parameters: impact Weber number $\left(\mathrm{We}=\rho D u^{2} / \sigma\right)$, impact Reynolds number $(\operatorname{Re}=\rho D u / \mu)$, and wetting angle $(\theta)$. Here $u$ and $D$ are the impact velocity and the initial drop diameter, while $\rho, \sigma$, and $\mu$ are the liquid density, surface tension and viscosity respectively. Fitting of the experimental data obtained for water droplet spreading diameter has been performed in [9]. Since the viscosity of glycerine is significantly higher than the viscosity of water, a glycerine droplet exhibits different behaviour when impacting on a flat surface. After the initial spreading, and achieving its maximum, the diameter of a glycerine droplet remains almost constant. The equation for fitting the spreading diameter of a glycerine droplet impacting on horizontal surfaces is obtained by modifying the equation for water droplet obtained in paper [9]. The remainder of the paper is organized in following manner: Section 2 presents numerical model and comparisons of experimental and fitted results. Section 3 presents comparisons between fitted and experimental data. Section 4 presents summary and conclusions.

\section{The most important properties of glycerine}

The spread of a single droplet of $85 \%$ glycerine is studied for three dry surfaces. There is a significant difference in dynamic viscosities of glycerine and water. On the other hand, their surface tensions are similar. Glycerine has been studied as a representative of liquids of high viscosity. Dynamic viscosity of glycerine is 116 time higher than that of water. Properties of glycerine are presented in the table below:

\begin{tabular}{|c|c|c|c|}
\hline & $\boldsymbol{\sigma}(\mathbf{N} / \mathbf{m})$ & $\boldsymbol{\mu}(\mathbf{m P a s})$ & $\boldsymbol{\rho}\left(\mathbf{k g} / \mathbf{m}^{\mathbf{3}}\right)$ \\
\hline Glycerine & 0,063 & 116 & 1220 \\
\hline
\end{tabular}

Table 1. Properties of glycerine

For experiments we used three different smooth surfaces: a smooth glass plate, a paraffin wax plate and a smooth PVC plate. The following figures (Fig. 1, Fig. 2 and Fig. 3) show desirable numerical behaviour of glycerine on these three surfaces. The only exception is glycerine with high Weber numbers ( $\mathrm{We}=802$ and $\mathrm{We}=1056)$ on wax.

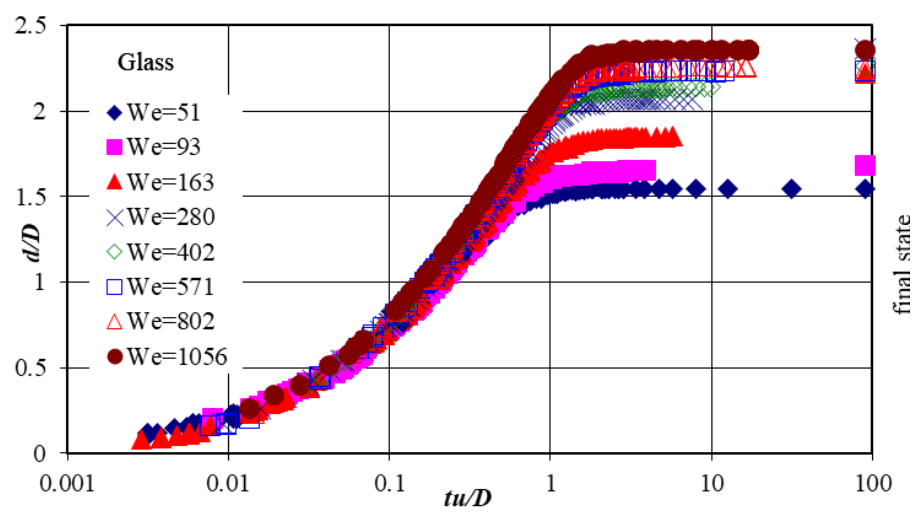

Fig. 1. Glycerine droplet impacting smooth glass

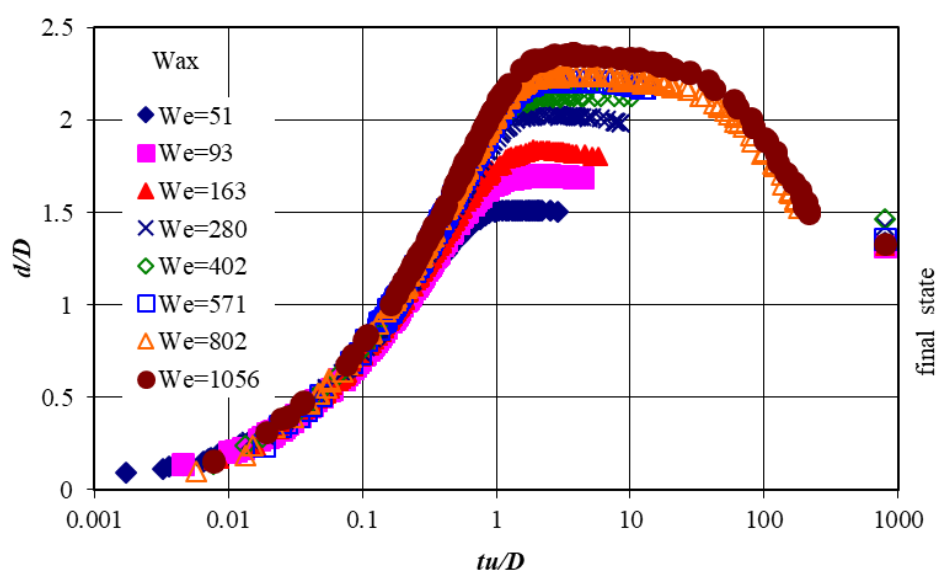

Fig. 2. Glycerine droplet impacting paraffin wax plate 


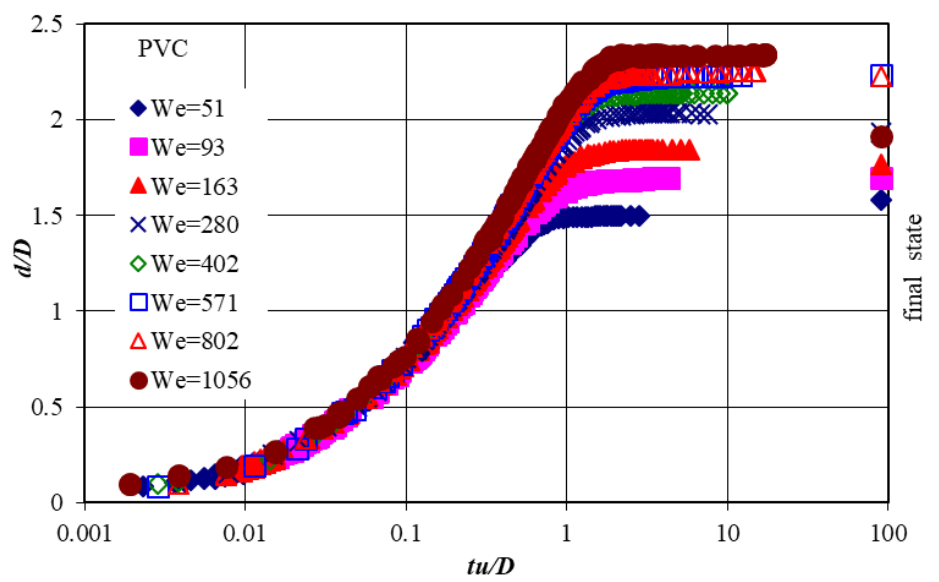

Fig. 3. Glycerine droplet impacting smooth PVC plate

\section{Numerical approach}

For the maximum spreading diameter we use the experimental data from [1] and [2]. Šikalo and Kostić in [9] have provided the rational approximation for water. The experimental data are fitted using rational and constant function given by

$$
x^{*}=\left\{\begin{array}{c}
\frac{0,125+10 t^{*}}{1+a t^{*}+b\left(t^{*}\right)^{2}}, \quad t \leq t^{*} \\
x_{\max }^{*}, \quad t>t^{*}
\end{array}\right.
$$

Because glycerine has 116 times higher viscosity than water, coefficients are modified. For glycerine, the function for fitting of the data will have two analytical expressions - the rational function and the constant function. Data for the impact of glycerine droplets, with diameter of $2.45 \mathrm{~mm}$, and the Weber numbers 51, 93, 163, 280, 402, 571, 802, 1056 are fitted to the combination of rational and constant functions. Coefficients of rational functions are: the first fixed coefficient 0.125 , because we assumed that the droplet deforms to $1 / 8$ of its original diameter before it starts spreading, where $t^{*}=0, x^{*}=0.125 D(x=0.125 D)$; the second fixed coefficient equal to 10 is linked to the initial speeding velocity of glycerine. The stationary point of the rational function (1) is the maximum of the experimental results, from which we determine the coefficients $a$ and $b$. According to the experimental data it is obtained $a>>b>0$. The quadratic trinomial $1+a t^{*}+b\left(t^{*}\right)^{2}$ has two real zeros because $a>>b$. All coefficients of quadratic trinomial are positive real numbers and zeroes of trinomial are negative. Function (1) is defined and differentiable for each $t^{*}>0$. Therefore:

$$
\left(x^{*}\right)^{\prime}=\frac{10\left[1+a t^{*}+b\left(t^{*}\right)^{2}\right]-\left(0.125+10 t^{*}\right)\left(a+2 b t^{*}\right)}{\left[1+a t^{*}+b\left(t^{*}\right)^{2}\right]^{2}}
$$

thus coefficients $a$ and $b$ are obtained from the condition

$$
\begin{aligned}
& 10\left[1+a t_{\text {max }}^{*}+b\left(t_{\text {max }}^{*}\right)^{2}\right]-\left(0.125+10 t_{\text {max }}^{*}\right)\left(a+2 b t_{\text {max }}^{*}\right)=0 \\
& x_{\text {max }}^{*}=\frac{0.125+10 t_{\text {max }}^{*}}{1+a t_{\text {max }}^{*}+b\left(t_{\text {max }}^{*}\right)^{2}}
\end{aligned}
$$

Equalities (2) and (3) imply

$$
a=\frac{10 t_{\max }^{*}-2 x_{\max }^{*}+0.25}{x_{\max }^{*} t_{\max }^{*}}
$$




$$
b=\frac{x_{\max }^{*}-0.125}{x_{\max }^{*}\left(t_{\max }^{*}\right)^{2}}
$$

Finally, we obtain

$$
x^{*}=\frac{0.125+10 t^{*}}{1+\frac{10 t_{\text {max }}^{*}-2 x_{\max }^{*}+0.25}{x_{\max }^{*} t_{\max }^{*}} t^{*}+\frac{x_{\text {max }}^{*}-0.125}{x_{\text {max }}^{*}\left(t_{\max }^{*}\right)^{2}}\left(t^{*}\right)^{2}}
$$

Numerical values of coefficients $a$ and $b$ for the glycerine droplet are given for different values of We $(51,93,163,280,402,571,802,1054)$ in Table 2 . The glycerine droplet impacts smooth, glass, paraffin wax and PVC. Coefficients $a$ and $b$ were calculated according to relations (4) and (5) given above.

\begin{tabular}{|c|c|c|c|c|}
\hline No & We & Re & $\boldsymbol{a}$ & $\boldsymbol{b}$ \\
\hline \multicolumn{5}{|c|}{ Glycerine - glass } \\
\hline 1. & 51 & 205 & 5,3338 & 0,3441 \\
\hline 2. & 93 & 190 & 5,5687 & 0,06 \\
\hline 3. & 163 & 179 & 5,0782 & 0,0281 \\
\hline 4. & 280 & 187 & 4,0742 & 0,1528 \\
\hline 5. & 402 & 197 & 4,3151 & 0,0346 \\
\hline 6. & 571 & 178 & 3,9841 & 0,0659 \\
\hline 7. & 802 & 167 & 4,1579 & 0,0185 \\
\hline 8. & 1056 & 157 & 3,5731 & 0,1184 \\
\hline
\end{tabular}

Table 2. The coefficients of the rational function using the experimental maximum for glass [4]

\begin{tabular}{|c|c|c|c|c|}
\hline No & We & Re & $\boldsymbol{a}$ & $\boldsymbol{b}$ \\
\hline \multicolumn{5}{|c|}{ Glycerine - wax } \\
\hline 1. & 51 & 205 & 4,9556 & 0,742 \\
\hline 2. & 93 & 190 & 4,9196 & 0,2437 \\
\hline 3. & 163 & 179 & 4,4398 & 0,2601 \\
\hline 4. & 280 & 187 & 4,0712 & 0,1976 \\
\hline 5. & 402 & 197 & 4,0499 & 0,1041 \\
\hline 6. & 571 & 178 & 3,7331 & 0,1617 \\
\hline 7. & 802 & 167 & 4,0777 & 0,0328 \\
\hline 8. & 1056 & 157 & 3,7281 & 0,068 \\
\hline
\end{tabular}

Table 3. The coefficients of the rational function using the experimental maximum for wax [4]

\begin{tabular}{|c|c|c|c|c|}
\hline No & We & Re & $\boldsymbol{a}$ & $\boldsymbol{b}$ \\
\hline \multicolumn{5}{|c|}{ Glycerine - PVC } \\
\hline 1. & 51 & 205 & 5,5904 & 0,3148 \\
\hline 2. & 93 & 190 & 5,4144 & 0,061 \\
\hline 3. & 163 & 179 & 4,6608 & 0,1542 \\
\hline 4. & 280 & 187 & 4,2756 & 0,1069 \\
\hline 5. & 402 & 197 & 4,0864 & 0,0923 \\
\hline 6. & 571 & 178 & 4,3046 & 0,0085 \\
\hline 7. & 802 & 167 & 4,1182 & 0,0283 \\
\hline 8. & 1056 & 157 & 3,3992 & 0,2005 \\
\hline
\end{tabular}

Table 4. The coefficients of the rational function using the experimental maximum for PVC [4]

The following figures illustrate the quality of approximations. 
31ST DAAAM INTERNATIONAL SYMPOSIUM ON INTELLIGENT MANUFACTURING AND AUTOMATION

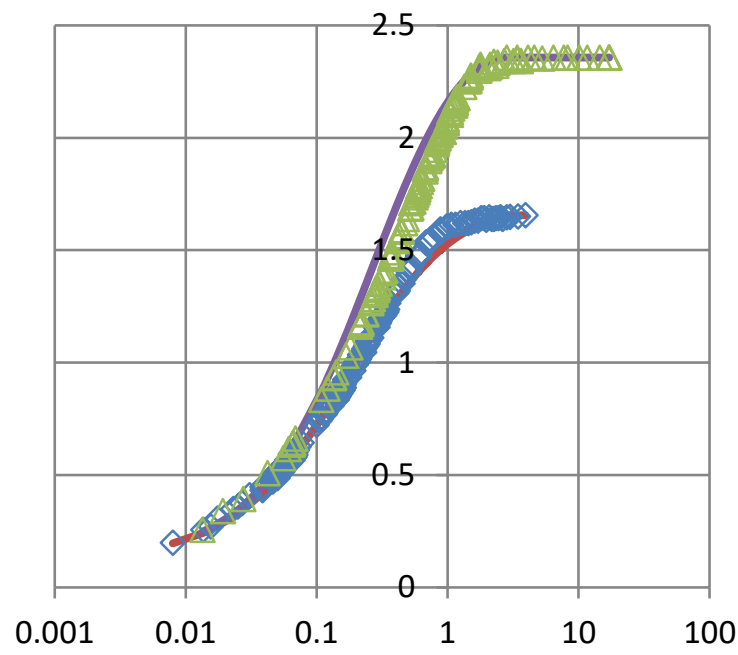

Fig. 4. Comparison of (Eq.6) with experimental data, $\mathrm{We}=93$ and $\mathrm{We}=1056$, of glycerine droplet impacting smooth glass

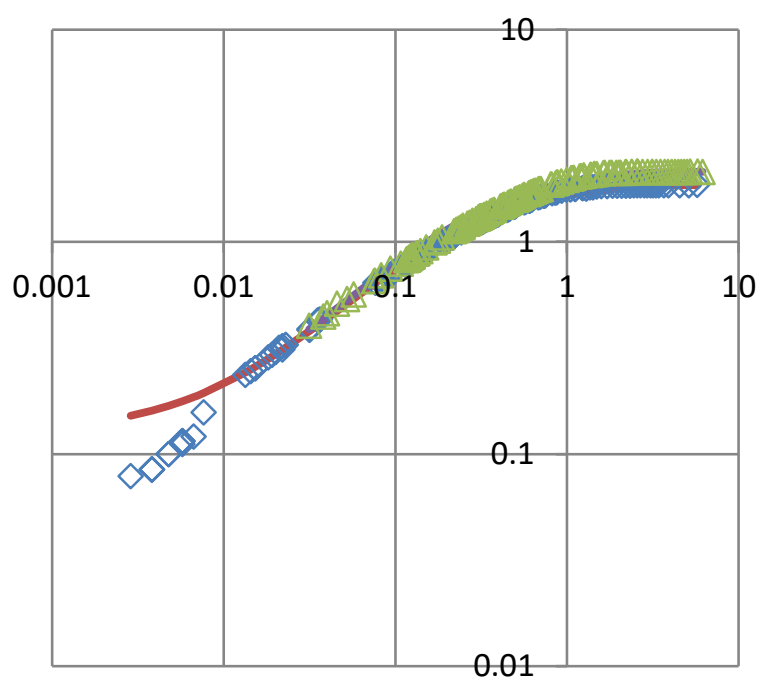

$\mathrm{We}=93$

Fitting, We $=93$

We $=1056$

Fitting, We $=1056$

Fig. 5. Comparison of (Eq.6) with experimental data, $\mathrm{We}=163$ and $\mathrm{We}=402$, of glycerine droplet impacting smooth glass

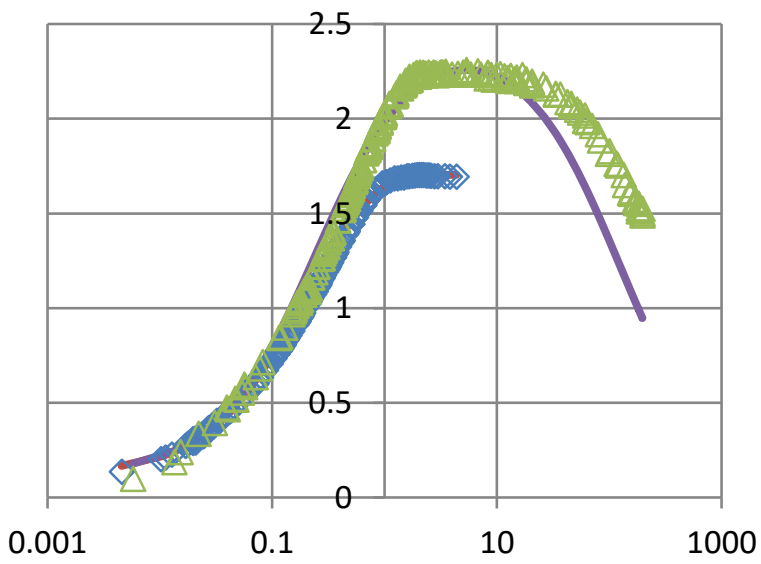

Fig. 6. Comparison of (Eq.6) with experimental data, $\mathrm{We}=93$ and $\mathrm{We}=802$, of glycerine droplet impacting paraffin wax

- $0947-$ 


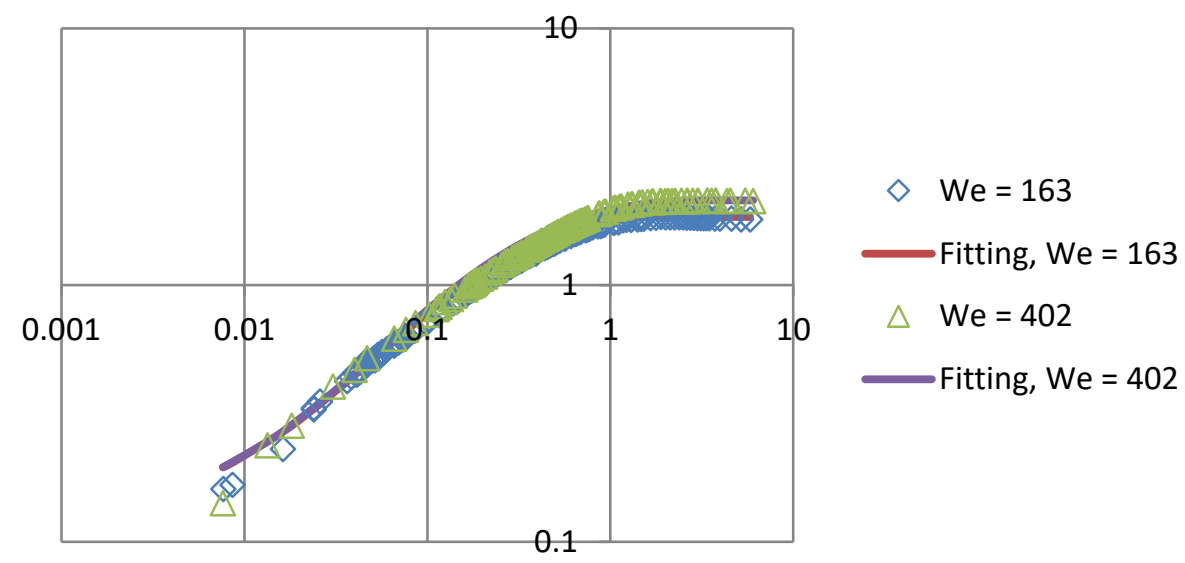

Fig. 7. Comparison of (Eq.6) with experimental data, $\mathrm{We}=163$ and $\mathrm{We}=402$, of glycerine droplet impacting paraffin wax

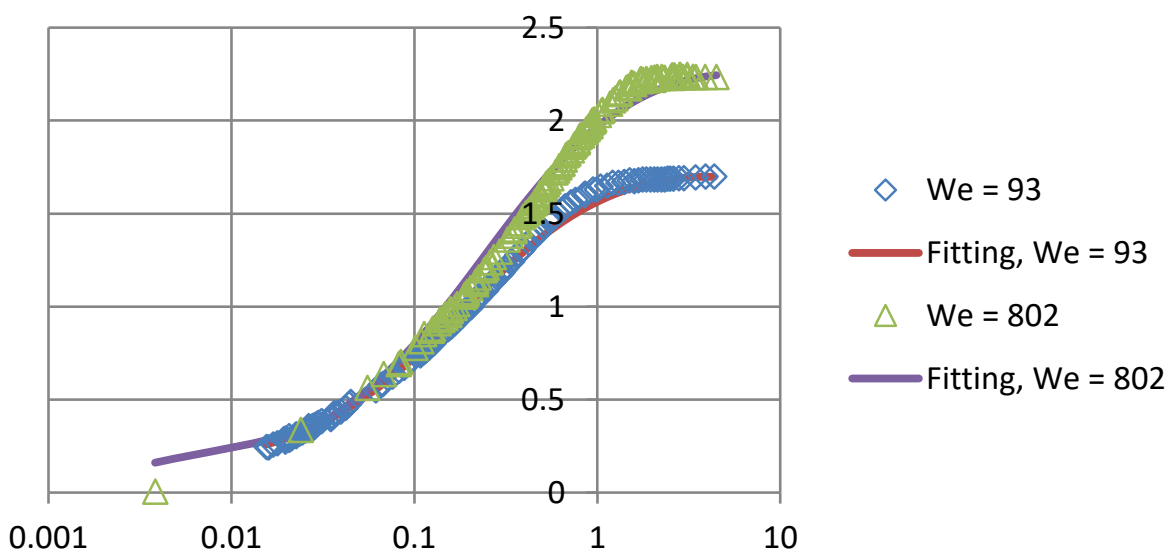

Fig. 8. Comparison of (Eq.6) with experimental data, $\mathrm{We}=93$ and $\mathrm{We}=802$, of glycerine droplet impacting PVC

\section{Conclusion}

In this paper, we considered the experimental data in the case when a drop of glycerine impacts onto the smooth glass, wax and plastic. We used some physical characteristics of droplets impacting a solid surface. The combination of the rational function and the constant function had a good match with the experimental data provided in the case of smooth glass and PVC plates. The proposed function in equation (6) approximates the experimental data well for smooth glass, Weber numbers range from 93 to 1056, and for all dimensionless time values $t^{*}\left(t^{*}=t u / D\right)$, as shown in Fig. 4. and Fig. 5.

Also, the approximation is very good for a droplet on a PVC substrate (Fig. 8), as well as for a droplet on a wax substrate for We < 402 (Fig. 7). In these cases viscous forces dominate. The maximum diameter of the spreading is not larger than the final one, therefore there is no droplet reducing. The equation (6) does not approximate the experimental data sufficiently accurate for a wax substrate, only for high We numbers, such as for We $=802$. That is the consequence of poor wetting (large wetting angles). In this case the maximum spreading diameter is larger than the final one and the capillary forces dominate, so that they reduce the droplet at the final spreading diameter. In this case the influence of the wetting angle should be included in the fitting equation. Further research will continue in that direction.

\section{References}

[1] Šikalo, Š., Marengo, M., Tropea, C., Ganić, E. N. (2002). Analysis of impact of droplets on horizontal surfaces, Experimental Thermal and Fluid Science, 25, pp. 503-510.

[2] Šikalo, S̆. (2003). Analysis of droplet impact onto horizontal and inclined surfaces, Shaker Verlag, Aachen, Germany.

[3] Šikalo, Š. and Ganić, E. N. (2006). Phenomena of Droplet-Surface Interactions, Experimental Thermal and Fluid Science, 31 (2), pp. 97-110. 
[4] Roisman, I.V., (2009). Inertia dominated drop collisions. II. An analytical solution of the Navier-Stokes equations for a spreading viscous film, Phys. Fluids, 21 (5) (2009) 052104.

[5] Šikalo, Š., Berberović, E., (2015). Analysis of Droplet Deposition in a Vertical Air-water Dispersed Flow, Procedia Engineering, 100, pp. 105-114.

[6] Berberović, E., Šikalo Š., (2015). Computational Modeling and Simulation of Nonisothermal Free-surface Flow of a Liquid Jet Impinging on a Heated Surface, Procedia Engineering, 100, pp. 115-124.

[7] Josserand, C., Thoroddsen, S. T. (2016). Drop impact on a solid surface, Annu. Rev. Fluid Mech. 48, $365-391$.

[8] Schremb, M., Borchert, S., Berberovic, E., Jakirlic, S., Roisman, I. V., Tropea, C. (2017). Computational modelling of flow and conjugate heat transfer of a drop impacting onto a cold wall. International Journal of Heat and Mass Transfer, Vol. 109, pp. 971-980.

[9] Šikalo, Š., Kostić, A., (2017). An equation for fitting spreading diameter of a liquid droplet impacting on flat surfaces, DEMI 2017, Proceedings of 13th International conference on Accomplishment in Mechanical and industrial Engineering, Banja Luka, pp. 237-242.

[10] M. Marengo, R. Rioboo, Š. Šikalo, C. Tropea 1998 Time evolution of drop spreading onto dry, smooth solid surfaces, Proceedings of the 14th International Conference on Liquid Atomization and Spray Systems - ILASSEurope '98, Manchester, UK, Ed. Andrew J. Yule.

[11] Šikalo, Š., Wilhelm, H. D., Roisman, I. V., Jakirlić, S., Tropea, C. (2005). Dynamic contact angle of spreading droplets: Experiments and simulations. Physics of Fluids, 17 (6), pp. 1-13. 